\title{
Automatic Fetal Heart Rate Monitoring in The Past, Present and Future
}

\author{
Kazuo Maeda* \\ Honorary professor, Obstetrics \& Gynecology, Tottori University, Japan
}

Received: May 31, 2018; Published: June 15, 2018

*Corresponding author: Kazuo Maeda, Honorary professor, Obstetrics \& Gynecology, Tottori University, 3-125 Nadamachi, Yonago, Tottoriken, 6830835, Japan

\begin{abstract}
Aims: To automatically diagnose intrapartum fetal heart rate (FHR).

Methods: Initial system was composed of HP2100A minicomputer with core memory, AD converter, puncher/reader of paper tape and Teletype keyboard/printer, where software was an expert's system diagnosing baseline tachy- or bradycardia, deceleration patterns and baseline variability. CTG data were punched paper tape and reproduced by its reader to analyze, due to poor capacity of core memory, which was only 4 kbytes. Next system was composed of microcomputer tips (MT-140) with small printer. Final one was a parallelcomputers for central system with 50 channel time sharing system, composed of experts knowledge, FHR score,which predict Apgar score, artificial neural network, which predict fetal outcome, and baseline frequency spectrum, which diagnose ominous sinusoidal FHR, which prepared doctor direct reporting system.
\end{abstract}

Results: Perinatal mortality reduced and cerebral palsy was zero, in the multiple system.

Future: Single parturient woman monitoring computer is planning, which includes FHR score, frequency spectrum and novel hypoxia index, by which infantile cerebral palsy is prevented, and direct reporting system.

Keywords: Fetus; Labor; Automatic Diagnosis; FHR; Computer; Past to Future

Abbreviations: FHR: Fetal Heart Rate; MT-140: Microcomputer Tips; HI: Hypoxia Index

\section{Introduction}

As FHR monitoring is mandatory in the labor management, its full operation during the labor process is troublesome, namely 10 or more hours continuous monitoring is recommended, where it is requested to watch the CTG chart continuously and diagnose unexpected FHR changes. That is a very hard work for attending doctor or staff. Thus, computerized FHR monitoring and prompt report of abnormal FHR change are requested, where the most correct diagnosis and correct expectation of fetal outcome are requested. Thus, the deceleration pattern classification [1] was the subject of computerized diagnosis in our dawn of computerized FHR diagnosis [2], where the baseline heart rate in $5 \mathrm{~min}$ was determined by counting FHR data in every $10 \mathrm{bpm}$ steps detecting the most data step, where FHR data were averaged and baseline FHR was determined, FHR variations were averaged to achieve long-term variability. Deceleration classification was complicated, i.e. dip-shape value was determined in every deceleration to classify $\mathrm{V}$ shape periodic and $U$ shape variable decelerations, then periodic one was divided into early and late decelerations by the lag time to uterine contraction [2]. However, as the character of late deceleration was vague and uncertain, the pattern classification method was collapsed in computerized monitoring, instead novel hypoxia index covered all decelerations and continuous bradycardia.

\section{Methods}

\section{FHR Score}

The FHR score was calculated by the sum of evaluation scores of the FHR baseline and the parts of decelerations by the author, 1969 [3] (Figure 1) and (Tables 1 \& 2), instead of subjective visual pattern classification. The fetus is abnormal, if FHR score is 10 or more, and highly abnormal if the score is 20 or more.

Results: Fetal outcome was predicted even in the 1st stage of labor as follows byregression equation. i.e. FHR score is useful to indicate early delivery in early stage of labor. 


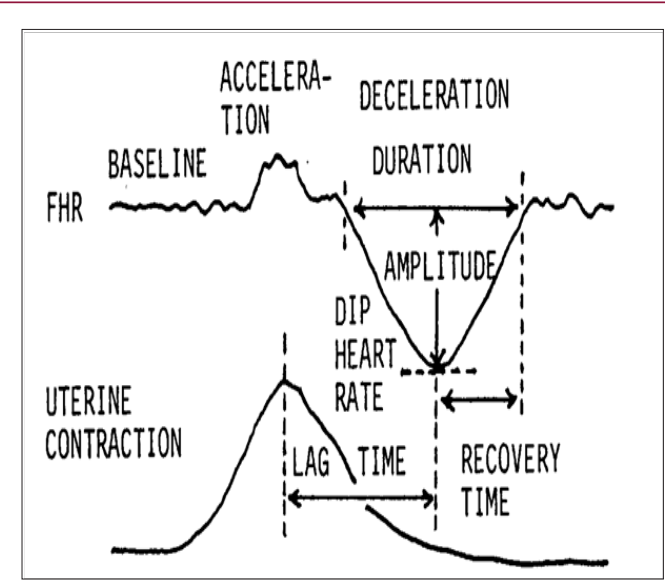

Figure 1: Determination of every parts of FHR changes in objective diagnosis offetal heart rate curve [3].

Table 1: Calculation of FHR score. Evaluation scores were summarized in 5 minutes achieving FHR score. Evaluation score was decided according to the percentage of low Apgar score lower than 7.

\begin{tabular}{|c|c|}
\hline FHR Signs & Evaluation Scores \\
\hline $\begin{array}{c}\text { FHR baseline } 119-130 \text { or } 160-180 \\
\text { bpm }\end{array}$ & 1 \\
\hline$<110$ or $>180$ bpm Deceleration & 3 \\
\hline Duration $>60$ sec & 3 \\
\hline Nadir $<100 \mathrm{bpm}$ & 2 \\
\hline Amplitude $>50 \mathrm{bpm}$ & 2 \\
\hline Recovery time $>40$ sec & 3 \\
\hline Lag time $>40$ sec & 3 \\
\hline No acceleration & 2 \\
\hline W-shaped & 4 \\
\hline
\end{tabular}

Table 2: Fetal outcome is predicted in the 1st stage of labor according to regression equations.

\begin{tabular}{|c|c|c|c|}
\hline FHR Score & Apgar Score & Asphyxia & UAph \\
\hline 10 & 6 & Mild & Normal \\
\hline 15 & 4 & Moderate & 7.07,Acidosis \\
\hline 20 & 3 & Severe & 6.98, Acidosis \\
\hline
\end{tabular}

\section{Hypoxia Index}

It is a novel FHR index to prevent cerebral palsy, created by the author.

Methods: As intrapartum hypoxic fetal brain damage was caused by the repetition of FHR decelerations, but not by late deceleration pattern, thus, deceleration duration as summarized and divided by the lowest FHR, which meant hypoxic intensity.

Hypoxia Index: Is the sum of deceleration durations (min) divided by the lowest FHR (bpm), and multiplied by 100 to be integer, where FHR was calculated instead of $\mathrm{PaO}_{2}$, because heart rate was parallel to $\mathrm{PaO}_{2}$ when $\mathrm{PaO}_{2}$ was lower than $50 \mathrm{mmHg}$ [4] and fetal $\mathrm{PaO} 2$ was lower than $50 \mathrm{mmHg}$ [3], while fetal blood sampling was hard in the labor. Hypoxia index covers all FHR decelerations including early, late and variable, as well as sudden and continuous bradycardia.

Results: Fetal hypoxia index (HI) was 25 or more in cases who had the loss of FHR variability and brain damage followed by infantile cerebral palsy, while the HI was 24 or less in the cases who had deceleration but neither fetal brain damage nor infantile cerebral palsy [5] (Table 3). Thus, hypoxia index is calculated in computerized fetal monitoring, warning the presence of FHR deceleration, hypoxia index at present and that safe hypoxia index is 24 or less, every 5 minutes.

Table 3: Chi square test of hypoxia index comparing cerebral palsy.

\begin{tabular}{|c|c|c|c|}
\hline Hypoxia Index & \multicolumn{2}{|c|}{ Cerebral Palsy } & Total \\
\hline & Yes & No & \\
\hline 25 or more & 6 & 0 & 6 \\
\hline 24 or less & 0 & 16 & 16 \\
\hline Total & 6 & 16 & 22 \\
\hline
\end{tabular}

\section{Frequency Spectrum} [6].

Frequency spectrum of FHR curves was studied by the authors

Method: Five minutes'FHR curve is always monitored by FHR score, hypoxia index and frequency spectrum, which reports the presence ofpathologic sinusoidal FHR when La/Ta ratio is 39 or more and at the same time the PPSD is 300 or more, because pathologic sinusoidal is very ominous by severe fetal anemia close to fetal death. Although there is another benign sinusoidal curve, which was caused by the synchronizlation of FHR and periodic fetal movements, e.g. fetal respiratory movments, but not an ominous sign, only diagnosed by the periodic fetal movements recorded by actocardiogram, where the false sinusoidal is not reported doctor, Thus the alarm of sinusoidal should not be overlooked in auomatic fetal monitoring, because pathologic sinusoidal FHR is always malignant and close to fetal death, thus urgent caesarean delivery followed by neonatal blood transfusion are performed to cure the baby from the demise.

\section{Discussion}

Fetal monitoring should be external method which is able to monitor the fetus before the rupture of membrane in the labor and even in pregnancy., namely, ultrasonic Doppler autocorrelation FHR meter, or abdominal fetal ECG with multiple electrodes excluding maternal ECG will detect FHR curve. FHR computer will be composed in single channel for single pregnant woman, or centralized multiple time sharing system monitoring multiple deliveries. Analyzing program will be the detection of basic FHR components, FHR score to predict fetal outcomes, hypoxia index to predict and prevent cerebral palsy, frequency spectrum analysis to diagnose pathologic sinusoidal heart rate and A/B ratio in actocardiogram, and so on. Quick and direct reporting system to doctor is mandatory for clinical purpose. Hypoxia Index (HI) is novel and unique in 
the present study to predict the loss of FHR baseline variability and fetal brain damage followed by cerebral palsy. The hypoxia index$=$ The sum of FHR deceleration duration ( $\mathrm{min}$ ) divided by the lowest FHR (bpm) and multiplied by 100, of which purpose is to collect the summation of hypoxic effects in repeated FHR decelerations, where 25 or more of $\mathrm{HI}$ predicts the cerebral palsy, and the HI is 24 or less to prevent cerebral palsy.The data of FHR curve frequency spectrum indicates pathologic sinusoidal FHRshowing fetal anemia [6]. A/B ratio is the ratio of FHR acceleration duration to that of fetal movement burst of which value is less than 1 when Apgar score is lower than [7]. The perinatal mortality decreased, and cerebral palsy was zero, when there was direct and rapid reporting system in centralized computer [8].

\section{Conclusion}

The computerized fetal monitoring improves busy obstetric doctors. Although thecaesarean delivery was allowed after the loss of FHR baseline variability in the common CTG, namely it prevented fetal death, while it was unable to prevent the cerebral palsy. Early delivery should be performed before the loss of variability to prevent fetal brain damage followed by cerebral palsy [5], where the hypoxia index is 24 or less in the update numeric diagnosis of intrapartum fetal monitoring, whereearly delivery is performed keeping the hypoxia index at 24 or less level to prevent the loss of variability and fetal brain damage followed by cerebral palsy.

\section{References}

1. Hon EH (1968) An Atlas of Fetal Heart Rate Patterns. In (Hon EH), (Eds.) Harty Press, New Haven.

2. Maeda K, Arima T, Tatsumura M, Nasgasawa T (1980) Computer-aided fetal heart rate analysis and automatic fetal-distress diagnosis during labor and pregnancy utilizing external technique in fetal monitoring. in (Maeda K, Arima T, Tatsumura M, Nasgasawa T), (Eds.) North-Holland Publishing Company, MEDINFO 80, Lindberg \& Kaihara, 1214-1218.

3. Maeda K, Kimura S, Nakano H (1969) Pathophysiology of Fetus. In (Maeda K, Kimura S, Nakano H), (Eds.) Fukuoka Printing, Fukuoka.

4. Umezawa J (1975) Studies on the relation between heart rate and $\mathrm{PaO2}$ in hypoxic rabbit: a comparative study for fetal heart rate change in labor. Acta Obstet Gynecol Jpn 28: 1203-1212.

5. Maeda K (2014) Modalities of fetal evaluation to detect fetal compromise prior to the development of significant neurological damage. JOGR 40: 2089-2094.

6. Maeda K, Nagasawa T (2005) Automatic computerized diagnosis of fetal sinusoidal heart rate. Fetal Duag Ther 20: 328-334.

7. Maeda K, Iwabe T, Ito T, Minagawa Y, Morokuma S et al. (2009) Detailed multigrade evaluation of fetal disorders with the quantified actocardiogram. J Perinat Med 37(4): 392-396.

8. Maeda K, Noguchi Y, Nagasawa T, Masaji Utsu, Fujihiko Matsumoto et al. (2012) Central computerized automatic fetal heart rate diagnosis with a rapid and direct alarm system. The Open Medical Devices Journal 4: $28-33$.

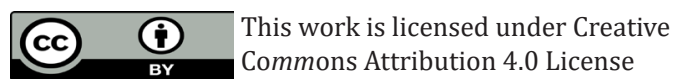

Submission Link: https://biomedres.us/submit-manuscript.php

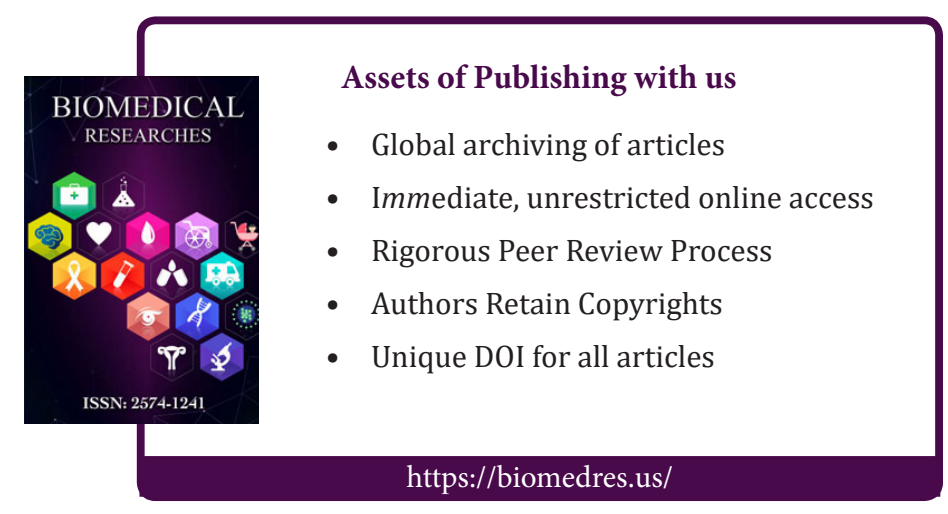

